\title{
LOCAL SPLITTING THEOREMS FOR RIEMANNIAN MANIFOLDS
}

\author{
M. CAI, G. J. GALLOWAY, AND Z. LIU
}

(Communicated by Christopher Croke)

\begin{abstract}
In this paper we establish two local versions of the Cheeger-Gromoll Splitting Theorem. We show that if a complete Riemannian manifold $M$ has nonnegative Ricci curvature outside a compact set $B$ and contains a line $\gamma$ which does not intersect $B$, then the line splits in a maximal neighborhood that is contained in $\overline{M \backslash B}$. We use this result to give a simplified proof that $M$ has a bounded number of ends. We also prove that if $M$ has sectional curvature which is nonnegative (and bounded from above) in a tubular neighborhood $U$ of a geodesic $\gamma$ which is a line in $U$, then $U$ splits along $\gamma$.
\end{abstract}

The Cheeger-Gromoll Splitting Theorem [CG] states that if a complete Riemannian manifold $M^{n}$ with nonnegative Ricci curvature contains a line $\gamma$, then $M^{n}$ is isometric to $\mathbb{R} \times N^{n-1}$ with the product metric. Here a line means a geodesic $\gamma$ defined on the interval $(-\infty, \infty)$ such that $d(\gamma(s), \gamma(t))=|s-t|$ for any $s$ and $t$. More recently Cheeger has suggested the problem of establishing some local version of the splitting theorem. The aim is to show that if the Ricci curvature is nonnegative in the "vicinity" of a given line then some region about the line splits. In this paper we consider two situations:

(1) The Ricci curvature is nonnegative outside a compact set disjoint from the line.

(2) The sectional curvature is nonnegative (and bounded from above) in a tubular neighborhood of the line.

Theorem 1 refers to the first situation, and Theorem 3 refers to the second situation. Theorem 2 is an application of Theorem 1. The more general situation in which the Ricci curvature is assumed nonnegative in a tubular neighborhood of the line remains open. The key difficulty in establishing a local splitting theorem stems from the fact that the region in which the curvature condition is assumed to hold is incomplete. Hence, arguments which routinely make use of the existence of minimal geodesic segments have to be modified or circumvented.

We would like to point out that the meaning of the phrase "local splitting" used here is different from that used by Anderson in his recent paper [A], where

Received by the editors June 10, 1992 and, in revised form, July 27, 1992.

1991 Mathematics Subject Classification. Primary 53C20.

Key words and phrases. Ricci curvature, line, asymptotic ray.

The second author was partially supported by NSF grant DMS- 9006678 . 
he deals with the topological splitting of tubular neighborhoods of minimal geodesics of finite length.

Theorem 1. Let $M^{n}$ be a complete Riemannian manifold with nonnegative Ricci curvature outside a compact set $B$. Suppose that $M^{n}$ contains a line $\gamma$ which does not intersect the compact set $B$. Then there is a closed subset $N \subset M$, such that $N$ is isometric to $\mathbb{R} \times V^{n-1}$ with the product metric, where the interior of $V^{n-1}$ is an (n-1)-dimensional submanifold. Moreover, for each $y \in \partial V$, $\mathbb{R} \times\{y\}$ intersects $\partial B$.

Intuitively, we can imagine that the line splits in a maximal (noncircular) tubular neighborhood outside the "bad set" $B$. When $B=\varnothing$, we have the original Cheeger-Gromoll Splitting Theorem.

The proof of Theorem 1 is contained in the following two lemmas.

Recall that given a ray $\gamma$ and a point $y \in M$, a ray $\sigma$ is called an asymptotic ray (or asymptote) from $y$ to $\gamma$ if there is a sequence of minimal geodesics $\sigma_{j}$ from $y$ to some $\gamma\left(t_{j}\right)$, with $t_{j} \rightarrow \infty$, such that $\sigma_{j}^{\prime}(0) \rightarrow \gamma^{\prime}(0)$.

Lemma 1. Let $M, B$, and $\gamma$ be as in Theorem 1. Let $\gamma^{ \pm}(t)=\gamma( \pm t), t \geq 0$. For any $x \in \gamma$, there is an open neighborhood $U_{x}$ of $x$, such that for all $y \in U_{x}$ any asymptotic ray from $y$ to either $\gamma^{+}$or $\gamma^{-}$does not intersect $B$.

Proof. Suppose the lemma is not true. Then for some $x=\gamma(a)$ we may assume there is a sequence $y_{j} \rightarrow x$ such that for each $j$ there is an asymptotic ray $\sigma_{j}$ from $y_{j}$ to $\gamma^{+}$which intersects $B$. Since $y_{j} \rightarrow x, \sigma_{j}$ has a subsequence $\nu_{j}$ converging to a ray $\sigma$ from $x$ (i.e., $\nu_{j}(0) \rightarrow \sigma(0)$ and $\left.\nu_{j}^{\prime}(0) \rightarrow \sigma^{\prime}(0)\right)$. Clearly, $\sigma$ intersects $B$. On the other hand, by the following sublemma, $\sigma$ coincides with $\left.\gamma\right|_{[a, \infty)}$ which is away from $B$, a contradiction.

Sublemma. Suppose $t \rightarrow \gamma(t), 0 \leq t<\infty$, is a ray. Assume for some $s>0$ there is a sequence of points $\left\{y_{j}\right\}$ converging to $\gamma(s)$ and a sequence of asymptotes $\left\{\sigma_{j}\right\}$ from $y_{j}$ to $\gamma$. Then any convergent subsequence $\nu_{j}$ of $\sigma_{j}$ converges to $\left.\gamma\right|_{[s, \infty)}$ (i.e., $\nu_{j}^{\prime}(0)$ converges to $\left.\gamma^{\prime}(s)\right)$.

Proof. Suppose a convergent subsequence, still denoted by $\sigma_{j}$, converges to $\sigma$. $\sigma$ is then a ray from $\gamma(s)$. To show $\sigma^{\prime}(0)=\gamma^{\prime}(s)$ as required, it suffices to show that

$$
d(\gamma(0), \sigma(1)) \geq d(\gamma(0), \gamma(s))+d(\gamma(s), \sigma(1))
$$

since the triangle inequality then implies equality.

For each $j$ there is a sequence of minimal geodesics $\sigma_{j i}$ from $y_{j}$ to $\gamma\left(t_{j i}\right)$ such that $t_{j i} \rightarrow \infty$ and $\sigma_{j i} \rightarrow \sigma_{j}$ as $i \rightarrow \infty$. Now it follows from the triangle inequality that

$$
\begin{aligned}
d\left(\gamma(0), \sigma_{j i}(1)\right) \geq & d\left(\gamma(0), \gamma\left(t_{j i}\right)\right)-d\left(\sigma_{j i}(1), \gamma\left(t_{j i}\right)\right) \\
= & d(\gamma(0), \gamma(s))+d\left(\gamma(s), \gamma\left(t_{j i}\right)\right) \\
& -\left[d\left(y_{j}, \gamma\left(t_{j i}\right)\right)-d\left(y_{j}, \sigma_{j i}(1)\right)\right] \\
\geq & d(\gamma(0), \gamma(s))+d\left(y_{j}, \sigma_{j i}(1)\right)-d\left(\gamma(s), y_{j}\right) .
\end{aligned}
$$

By letting first $i \rightarrow \infty$ and then $j \rightarrow \infty$, we obtain the inequality (*). This completes the proof of the sublemma and, in turn, Lemma 1.

Lemma 2. Let $\gamma$ be a line in a complete Riemannian manifold $M^{n}$. Suppose that there exists a neighborhood $U$ of $\gamma(0)$ such that the Ricci curvature of $M$ 
is nonnegative along asymptotes emanating from points in $U$. Then there is a tubular neighborhood $V^{n}$ of $\gamma$ which is isometric to $\mathbb{R} \times V_{0}^{n-1}$. Moreover, each geodesic $\gamma_{x}=\mathbb{R} \times\{x\}, x \in V_{0}^{n-1}$, is a line in $M$.

The proof of this lemma draws on elements from various papers (see, e.g., [CG, E, EH, G1, G2]). In the proof, we occasionally make use of the following basic properties of Busemann functions: Let $\gamma$ be a ray and $b=b_{\gamma}$ its associated Busemann function. Then:

(a) $|b(x)-b(y)| \leq d(x, y)$, and

(b) along an asymptote $\eta$ to $\gamma, b(\eta(t))=b(\eta(0))+t$.

Proof of Lemma 2. Let $b^{ \pm}$be the Busemann functions associated to the line $\gamma$. As follows from [EH, Remark 1, p. 146] (cf. also [G1, Theorem 2]), $b^{ \pm}$and hence $B=b^{+}+b^{-}$are continuous subharmonic functions on $U$. Since $B \leq 0$ and $B(\gamma(0))=0, B \equiv 0$ on $U$ by the maximum principle for subharmonic functions. It follows as usual that $b^{+}$and $b^{-}$are $C^{\infty}$ harmonic functions on $U$ with $\left\|\nabla b^{ \pm}\right\|=1$.

Let $V_{0}=U \cap\left\{b^{+}=0\right\}$. Note that $b^{-}=0$ along $V_{0}$. For each $x \in V_{0}$, let $\sigma_{x}^{+}:[0, \infty) \rightarrow M$ be a unit speed asymptote to $\gamma^{+}$from $x$. We observe that $\sigma_{x}^{+}$is a $V_{0}$-ray, i.e., for all $t \geq 0$,

$$
L\left(\left.\sigma_{x}^{+}\right|_{[0, t]}\right)=d\left(V_{0}, \sigma_{x}^{+}(t)\right) .
$$

Indeed, we have for any $y \in V_{0}$

$$
d\left(y, \sigma_{x}^{+}(t)\right) \geq b^{+}\left(\sigma_{x}^{+}(t)\right)-b^{+}(y)=b^{+}(x)+t-b^{+}(y)=t=L\left(\left.\sigma_{x}^{+}\right|_{[0, t]}\right),
$$

where $L$ denotes arc length. Hence, $\sigma_{x}^{+}$(and similarly $\sigma_{x}^{-}$) is a $V_{0}$-ray, and in particular, $\sigma_{x}^{ \pm}$meet $V_{0}$ orthogonally. It follows that there are no focal points to $V_{0}$ along its normal geodesics. Moreover, asymptotes from distinct points in $V_{0}$ do not intersect. From these facts it easily follows that the normal exponential map along $V_{0}$,

$$
\Phi: \mathbb{R} \times V_{0} \rightarrow M, \quad \Phi(t, x)=\exp _{x}(t N),
$$

where $N=\left.\nabla b^{+}\right|_{V_{0}}$ is a diffeomorphism onto its image $V=\Phi\left(\mathbb{R} \times V_{0}\right)$. Note by assumption that the Ricci curvature on $V$ is nonnegative. It is now standard that the unit tangent vector field to the normal geodesics $N=\Phi_{*}(\partial / \partial t)$ is parallel and hence that $\Phi$ is an isometry. Indeed, the mean curvature $H=H_{t}$ of $V_{t}=\Phi\left(\{t\} \times V_{0}\right)$ obeys

$$
\frac{\partial H}{\partial t}=\operatorname{Ric}(N, N)+\|\nabla N\|^{2} \geq \frac{1}{n-1} H^{2} .
$$

It follows easily from this differential inequality that unless $\nabla N \equiv 0$ on $V, H_{t}$ must blow up in finite time along some normal geodesic.

Finally, we show that each normal geodesic $t \rightarrow \sigma_{x}(t):=\Phi(t, x)$ is a line in $M$. Indeed, we have, for arbitrary $t_{1}<0<t_{2}$,

$$
\begin{aligned}
& d\left(\sigma_{x}\left(t_{1}\right), \sigma_{x}\left(t_{2}\right)\right) \geq b^{+}\left(\sigma_{x}\left(t_{2}\right)\right)-b^{+}\left(\sigma_{x}\left(t_{1}\right)\right) \\
& \quad \geq b^{+}\left(\sigma_{x}\left(t_{2}\right)\right)+b^{-}\left(\sigma_{x}\left(t_{1}\right)\right)=b^{+}\left(\sigma_{x}^{+}\left(t_{2}\right)\right)+b^{-}\left(\sigma_{x}^{-}\left(-t_{1}\right)\right) \\
& \left.\quad=\left(b^{+}(x)+t_{2}\right)\right)+\left(b^{-}(x)+\left(-t_{1}\right)\right)=t_{2}+\left(-t_{1}\right)=L\left(\left.\sigma_{x}\right|_{\left[t_{1}, t_{2}\right]}\right),
\end{aligned}
$$

where we have used $b^{+}=b^{-}=0$ along $V_{0}$. This completes the proof of Lemma 2. 
Proof of Theorem 1. Lemmas 1 and 2 imply that a tubular neighborhood $V$ of $\gamma$ splits isometrically as $V_{0} \times \gamma$ with the product metric, where $b^{+}=b^{-}=0$ along $V_{0}$. Suppose $y \in \partial V_{0}$ is a boundary point. Take a continuous curve $\tau(s), 0 \leq s \leq S$, from $\gamma(0)$ to $y$ in $\overline{V_{0}}$ with $\tau([0, S)) \subset V_{0}$. Let $v(s)=$ $\nabla b^{+}(\tau(s)), 0 \leq s<S$. In the unit tangent bundle, take the limit of $v(s)$, $v=\lim _{s \rightarrow S} v(s)$. By the continuity of the exponential map, $\sigma_{y}(t):=\exp _{y}(t v)=$ $\lim _{s \rightarrow S} \exp _{\tau(s)}(t v(s))$. Moreover, since $b^{ \pm}\left(\sigma_{x}( \pm t)\right)=t, t \geq 0$, continue to hold after taking limits, we can apply the argument at the end of the proof of Lemma 2 to conclude that $t \rightarrow \exp _{y}(t v)$ is also a line.

Now the theorem follows by taking $N$ to be the closure of a maximal open set $U$, the union of all splittings described in Lemmas 1 and 2 . If the limiting line described above does not intersect $B$, then we can apply Lemmas 1 and 2 to this limiting line, and it splits in a tubular neighborhood of its own, contradicting the maximality of $U$. This completes the proof of Theorem 1 .

Cai [C], Li and Tam [LT], and Liu [L] have given independent proofs that a complete Riemannian manifold with nonnegative Ricci curvature outside a compact set $K$ has finitely many ends. (They also obtain upper bounds for the number of ends in terms of the lower bound on the Ricci curvature and the diameter of $K$.) This result generalizes the well-known consequence of the Cheeger-Gromoll Splitting Theorem that a complete Riemannian manifold with everywhere nonnegative Ricci curvature has at most two ends.

We indicate how Theorem 1 can be used to simplify the proof in [C] and relate it more directly to the aforementioned application of the Cheeger-Gromoll Splitting Theorem.

Theorem 2 [C, LT, L]. If $M^{n}$ is a complete Riemannian manifold with Ricci curvature nonnegative outside a compact set $K$, then $M$ has finitely many ends.

Proof. Let $B_{o}(a)$ be a ball of radius $a$ centered at $o$ which contains $K$. Suppose that $\gamma$ is a line in $M \backslash B_{o}(a)$. Let $B=b^{+}+b^{-}$, where $b^{ \pm}$are the Busemann functions associated to $\gamma$.

Claim. $B(o) \geq-2 a$.

Proof of Claim. Let $\sigma:[0, l] \rightarrow M$ be a shortest geodesic from $\gamma$ to $\partial B_{o}(a) . \sigma$ meets $\gamma$ orthogonally. Moreover, since the function $B$ is invariant under parameter translation of $\gamma$, we can assume $\sigma(0)=\gamma(0)$. Now apply Theorem 1 with $B=B_{o}(a)$. Since $V^{n-1}$ is totally geodesic, $\sigma(t) \in V^{n-1}$, for all $t \in[0, l]$. Thus $B(\sigma(t))=0, \forall t \in[0, l]$. In particular, $B(p)=0$ where $p=\sigma(l) \in \partial B_{o}(a)$. Now, for all $x, y \in M,|B(x)-B(y)| \leq 2 d(x, y)$. Hence,

$$
|B(o)|=|B(o)-B(p)| \leq 2 d(o, p)=2 a,
$$

which proves the claim.

Now suppose $M$ has infinitely many ends. Then there are infinitely many distinct rays in $M$ emanating from $o$. Hence clearly there exist rays $\gamma_{1}, \gamma_{2}$ from $O$ which satisfy

$$
d\left(\gamma_{1}(4 a), \gamma_{2}(4 a)\right) \leq 2 a .
$$

The argument on p. 376 in [C] then shows how to construct a line $\gamma$ in $M \backslash B_{o}(a)$ which satisfies

$$
d\left(\gamma(t), B_{o}(a)\right) \geq|t|+2 a \quad \forall t \in \mathbb{R}
$$


Rewriting this we have

$$
|t|-d(o, \gamma(t)) \leq-3 a .
$$

Letting $t \rightarrow \pm \infty$ we obtain $b^{ \pm}(o) \leq-3 a$, and hence $B(o) \leq-6 a$, contradicting the claim. This proves Theorem 2 .

In the following theorem we establish the splitting of a tubular neighborhood of a line under the assumption that the sectional curvature is nonnegative (and bounded from above) in this neighborhood.

Theorem 3. Let $\gamma$ be a geodesic in a complete Riemannian manifold $M^{n}$, and let $U=\{x \in M: d(x, \gamma)<\epsilon\}$ be a fixed tubular neighborhood of $\gamma$. If the sectional curvature of $M$ is nonnegative and bounded from above in $U$ and $\gamma$ is a line in $U$, i.e., $L\left(\left.\gamma\right|_{[s, t]}\right)$ is minimal among all curves in $U$ connecting $\gamma(s)$ and $\gamma(t)$, then $U$ splits isometrically into a product $\mathbb{R} \times U^{\prime}$.

Remark. Let $d_{U}$ denote the distance function in $U$, viewed as a Riemannian manifold in its own right. We would like to point out that, for any $p \in U$, $d_{U}(p, \gamma)=d_{M}(p, \gamma)$. Indeed, for any $\delta$ with $d_{M}(p, \gamma)<\delta<\epsilon$, there is a $q$ on $\gamma$ such that $d_{M}(p, q)<\delta$. Let $\mu$ be a minimal geodesic in $M$ from $p$ to $q$. Clearly $\mu$ must stay in $U$ or else $L(\mu)>\epsilon$ by the definition of $U$. This implies that $d_{U}(p, \gamma) \leq L(\mu)<\delta$, which proves that $d_{U}(p, \gamma) \leq d_{M}(p, \gamma)$. On the other hand, $d_{U} \geq d_{M}$. Our assertion follows.

Throughout the proof of Theorem 3, we write $d$ for $d_{U}$, unless otherwise indicated.

Proof. We will prove Theorem 3 assuming $\epsilon$ is less than or equal to $\pi / 2 \sqrt{K_{0}}$, where $K_{0}$ is an upper bound on the sectional curvature of $M$ in $U$. Extension to arbitrary $\epsilon$ follows from a straightforward continuation argument similar to the one used in Theorem 1.

Fix $p \in U$. Since $\gamma$ is a line in $U$, the function $t \rightarrow d(p, \gamma(t))$ achieves a minimum at some $t=t_{0}$. Hence by the above remark, we can find a minimal geodesic $\mu:\left[0, s_{0}\right] \rightarrow M$ from $\mu(0)=\gamma\left(t_{0}\right)$ to $\mu\left(s_{0}\right)=p$ contained in $U$.

Let $t \rightarrow N(t)$ be the parallel translate of $\mu^{\prime}(0)$ along $\gamma$. Consider the 2parameter map $\beta:\left[0, s_{0}\right] \times \mathbb{R} \rightarrow M^{n}$ defined by

$$
\beta(s, t)=\exp _{\gamma(t)}(s N) \text {. }
$$

Note that $\beta\left(\left[0, s_{0}\right] \times \mathbb{R}\right) \subset U$. Let $(s, t) \rightarrow J(s, t)$ and $(s, t) \rightarrow N(s, t)$ be the vector fields along $\beta$ defined by

$$
N(s, t)=\frac{\partial \beta}{\partial s}(s, t) \text { and } J(s, t)=\frac{\partial \beta}{\partial t}(s, t) .
$$

Claim 1. $\|J(s, t)\| \leq 1, \forall(s, t) \in\left[0, s_{0}\right] \times \mathbb{R}$.

Proof. For each $t, s \rightarrow J(s, t)$ is a Jacobi field along the geodesic $s \rightarrow \beta(s, t)$ satisfying the initial conditions

$$
\begin{aligned}
& J(0, t)=\frac{\partial \beta}{\partial t}(0, t)=\gamma^{\prime}(t), \\
& \frac{D J}{\partial s}(0, t)=\frac{D N}{\partial t}(0, t)=0 .
\end{aligned}
$$

Moreover, by the choice of $\epsilon$ and the Rauch comparison theorem (version II, cf. [CE]), each geodesic $s \rightarrow \beta(s, t)$ is focal point-free on $\left[0, s_{0}\right]$ relative to the 
geodesic hypersurface based at $\gamma(t)$ and orthogonal to $N(0, t)$. Claim 1 now follows from the assumption of nonnegative curvature and a second application of the Rauch comparison theorem.

For each positive integer $n$, consider the curve segments $\sigma_{n}^{ \pm}:\left[0, n \mp t_{0}\right] \rightarrow$ $M$ defined by

$$
\begin{array}{ll}
\sigma_{n}^{+}(t)=\beta\left(s_{0}-\frac{s_{0}}{n-t_{0}} t, t_{0}+t\right), & 0 \leq t \leq n-t_{0}, \\
\sigma_{n}^{-}(t)=\beta\left(s_{0}-\frac{s_{0}}{n+t_{0}} t, t_{0}-t\right), & 0 \leq t \leq n+t_{0} .
\end{array}
$$

For each $n, \sigma_{n}^{ \pm}$is contained in $U$ and extends from $p$ to $\gamma( \pm n)$. We do not know a priori that these are minimal segments. However, we now show that they are at least limit minimizing.

Claim 2. The segments $\left\{\sigma_{n}^{ \pm}\right\}$are limit minimizing, i.e.,

$$
\lim _{n \rightarrow \infty} L\left(\sigma_{n}^{ \pm}\right)-d(p, \gamma( \pm n))=0 .
$$

Comment. An analogous notion of limit maximizing timelike curves has been used in Lorentzian geometry (cf. [BE, EG]).

Proof of Claim 2. We estimate the length of $\sigma_{n}^{+}$. Using Claim 1 and the fact that $N$ and $J$ are orthogonal, we obtain

$$
\begin{aligned}
\left\|\frac{d o_{n}^{+}}{d t}\right\| & =\left\|\frac{-s_{0}}{n-t_{0}} N+J\right\|=\sqrt{\frac{s_{0}^{2}}{\left(n-t_{0}\right)^{2}}+\|J\|^{2}} \\
& \leq \sqrt{\frac{s_{0}^{2}}{\left(n-t_{0}\right)^{2}}+1}=\frac{\sqrt{s_{0}^{2}+\left(n-t_{0}\right)^{2}}}{n-t_{0}} .
\end{aligned}
$$

Hence

$$
L\left(\sigma_{n}^{+}\right)=\int_{0}^{n-t_{0}}\left\|\frac{d \sigma_{n}^{+}}{d t}\right\| d t \leq \sqrt{s_{0}^{2}+\left(n-t_{0}\right)^{2}}
$$

Similarly,

$$
L\left(\sigma_{n}^{-}\right) \leq \sqrt{s_{0}^{2}+\left(n+t_{0}\right)^{2}} .
$$

Adding these inequalities we obtain

$$
\begin{aligned}
L\left(\sigma_{n}^{+}\right)+L\left(\sigma_{n}^{-}\right) \leq & \sqrt{s_{0}^{2}+\left(n-t_{0}\right)^{2}}+\sqrt{s_{0}^{2}+\left(n+t_{0}\right)^{2}} \\
= & 2 n+\left(\sqrt{s_{0}^{2}+\left(n-t_{0}\right)^{2}}-n\right)+\left(\sqrt{s_{0}^{2}+\left(n+t_{0}\right)^{2}}-n\right) \\
= & d(\gamma(-n), \gamma(n))+\frac{s_{0}^{2}+t_{0}^{2}-2 n t_{0}}{\sqrt{s_{0}^{2}+\left(n-t_{0}\right)^{2}}+n} \\
& +\frac{s_{0}^{2}+t_{0}^{2}+2 n t_{0}}{\sqrt{s_{0}^{2}+\left(n+t_{0}\right)^{2}}+n} \\
\leq & d(p, \gamma(-n))+d(p, \gamma(n))+\frac{2 s_{0}^{2}+2 t_{0}^{2}}{2 n-\left|t_{0}\right|} .
\end{aligned}
$$


Thus,

$$
\left(L\left(\sigma_{n}^{+}\right)-d(p, \gamma(n))\right)+\left(L\left(\sigma_{n}^{-}\right)-d(p, \gamma(-n))\right) \leq \frac{2 s_{0}^{2}+2 t_{0}^{2}}{2 n-\left|t_{0}\right|} .
$$

Since the terms in brackets are nonnegative, letting $n \rightarrow \infty$ yields the claim.

It follows from Claim 2 that the sequences $\left\{\sigma_{n}^{ \pm}\right\}$are, in fact, limit minimizing on each fixed parameter interval $[0, a], a>0$, i.e.,

$$
\lim _{n \rightarrow \infty} L\left(\left.\sigma_{n}^{ \pm}\right|_{[0, a]}\right)-d\left(p, \sigma_{n}^{ \pm}(a)\right)=0
$$

Indeed, the triangle inequality implies

$$
L\left(\left.\sigma_{n}^{ \pm}\right|_{[0, a]}\right)-d\left(p, \sigma_{n}^{ \pm}(a)\right) \leq L\left(\sigma_{n}^{ \pm}\right)-d(p, \gamma( \pm n))
$$

Now consider the curves $\sigma^{ \pm}:[0, \infty) \rightarrow M$ in $U$ defined by

$$
\sigma^{ \pm}(t)=\beta\left(s_{0}, t_{0} \pm t\right)
$$

Claim 3. $\sigma^{ \pm}$, when parametrized with respect to arc length, are rays emanating from $p$.

Proof. The sequences $\left\{\sigma_{n}^{ \pm}\right\}$converge smoothly to $\sigma^{ \pm}$, and for each $a>0$

$$
L\left(\left.\sigma^{ \pm}\right|_{[0, a]}\right)=\lim _{n \rightarrow \infty} L\left(\left.\sigma_{n}^{ \pm}\right|_{[0, a]}\right)=\lim _{n \rightarrow \infty} d\left(p, \sigma_{n}^{ \pm}(a)\right)=d\left(p, \sigma^{ \pm}(a)\right) .
$$

Since, by the triangle inequality, $d\left(p, \sigma^{ \pm}(a)\right) \rightarrow \infty$ as $a \rightarrow \infty$, the claim follows.

Although the parameter $t$ along $\sigma^{ \pm}$must turn out to be arc length, we do not use this fact in the proof. Let $\hat{\sigma}^{ \pm}:[0, \infty) \rightarrow M$ denote $\sigma^{ \pm}$parametrized in terms of arc length $u=f^{ \pm}(t) . \quad \hat{\sigma}^{ \pm}$are rays emanating from $p$ which arise as the limit of limit minimizing curve segments. We refer to such rays as generalized asymptotes. Generalized asymptotes enjoy many of the properties of ordinary asymptotes. In particular, we have

Claim 4. The functions $b_{p, u}^{ \pm}: U \rightarrow \mathbb{R}(p \in U, u>0)$ introduced with respect to asymptotes in $[\mathrm{EH}]$ and defined by

$$
b_{p, u}^{ \pm}(x)=b^{ \pm}(p)+u-d\left(x, \hat{\sigma}^{ \pm}(u)\right)
$$

are lower support functions for the Busemann functions $b^{ \pm}$associated to the line $\gamma$ (and defined with respect to the distance function $d_{U}$ ).

Proof. Clearly $b_{p, u}^{ \pm}(p)=b^{ \pm}(p)$. We must show that $b_{p, u}^{ \pm}(x) \leq b^{ \pm}(x)$ for $x \in U$. Let

$$
\epsilon_{n}^{ \pm}=L\left(\sigma_{n}^{ \pm}\right)-d(p, \gamma( \pm n)) .
$$

Then for each $t>0$ and $n$ sufficiently large

$$
\begin{aligned}
d(x, \gamma( \pm n)) & =d\left(x, \sigma_{n}^{ \pm}\left(n \mp t_{0}\right)\right) \\
& \leq d\left(x, \sigma_{n}^{ \pm}(t)\right)+d\left(\sigma_{n}^{ \pm}(t), \sigma_{n}^{ \pm}\left(n \mp t_{0}\right)\right) \\
& \leq d\left(x, \sigma_{n}^{ \pm}(t)\right)+L\left(\left.\sigma_{n}^{ \pm}\right|_{\left[t, n \mp t_{0}\right]}\right) \\
& =d\left(x, \sigma_{n}^{ \pm}(t)\right)+L\left(\sigma_{n}^{ \pm}\right)-L\left(\left.\sigma_{n}^{ \pm}\right|_{[0, t]}\right) \\
& =d\left(x, \sigma_{n}^{ \pm}(t)\right)+d(p, \gamma( \pm n))+\epsilon_{n}-L\left(\left.\sigma_{n}^{ \pm}\right|_{[0, t]}\right) .
\end{aligned}
$$

Hence,

$$
n-d(x, \gamma( \pm n)) \geq n-d(p, \gamma( \pm n))+L\left(\left.\sigma_{n}^{ \pm}\right|_{[0, t]}\right)-\epsilon_{n}-d\left(x, \sigma_{n}^{ \pm}(t)\right) .
$$


Letting $n \rightarrow \infty$, we obtain

$$
b^{ \pm}(x) \geq b^{ \pm}(p)+L\left(\left.\sigma^{ \pm}\right|_{[0, t]}\right)-d\left(x, \sigma^{ \pm}(t)\right),
$$

which implies

$$
b^{ \pm}(x) \geq b^{ \pm}(p)+u-d\left(x, \hat{\sigma}^{ \pm}(u)\right),
$$

for each $u>0$, as desired.

Comment. Setting $x=\hat{\sigma}^{ \pm}(v), v<u$, in $(*)$ and using the triangle inequality to establish the reverse inequality, we obtain for generalized asymptotes $\hat{\sigma}^{ \pm}$

$$
b^{ \pm}\left(\hat{\sigma}^{ \pm}(u)\right)=b^{ \pm}(p)+u, \quad u \geq 0 .
$$

The proof of Theorem 3 now proceeds along familiar lines. We have $b^{+}+$ $b^{-} \leq 0$ on $U$ with equality along $\gamma$. Moreover, $b^{ \pm}$and hence $b^{+}+b^{-}$ are subharmonic (in fact, convex) on $U$ in the sense of support functions. This uses the fact that since $d\left(p, \hat{\sigma}^{ \pm}(u)\right)$ is realized by a minimal geodesic in $U$, by the continuity of the exponential map, so is $d\left(x, \hat{\sigma}^{ \pm}(u)\right)$ for any $x$ sufficiently close to $p$ after $u$ is fixed. Hence the proof of subharmonicity remains unchanged. It follows (cf. the proof of Lemma 2) that $b^{+}$and $b^{-}$are $C^{\infty}$ harmonic functions on $U$ with $\left\|\nabla b^{ \pm}\right\|=1$. In fact, at each point of $U$, $\nabla b^{ \pm}$must coincide with a tangent vector to some generalized asymptote $\hat{\sigma}^{ \pm}$. The standard evolution equation (cf. $[\mathrm{CG}, \mathrm{EH}]$ )

$$
X\left(\Delta b^{+}\right)=-\operatorname{Ric}(X, X)-\|\nabla X\|^{2}
$$

shows that $X=\nabla b^{+}$is parallel on $U$. Since the integral curves of $\nabla b^{+}$ coincide with the generalized asymptotes $\hat{\sigma}^{+}$(and hence are complete in $U$ ), normally exponentiating off $b^{+}=0$ gives the desired splitting of $U$. Theorem 3 is proved.

We suspect that the assumption of an upper curvature bound in Theorem 3 is unnecessary, i.e., that it is merely an artifact of our proof method. In an earlier version of this paper we omitted this assumption. We thank S. Zhu for bringing this oversight to our attention.

\section{REFERENCES}

[A] M. Anderson, Hausdorff perturbations of Ricci flat manifolds and the splitting theorem, Duke Math. J. 68 (1992), 67-82.

[BE] J. K. Beem and P. E. Ehrlich, Global Lorentzian geometry, Pure Appl. Math., vol. 67, Dekker, New York, 1981.

[C] M. Cai, Ends of Riemannian manifolds with nonnegative Ricci curvature outside a compact set, Bull. Amer. Math. Soc. (N.S.) 24 (1991), 371-377.

[CE] J. Cheeger and D. G. Ebin, Comparison theorems in Riemannian geometry, North-Holland, Amsterdam, 1975.

[CG] J. Cheeger and D. Gromoll, The splitting theorem for manifolds of nonnegative Ricci curvature, J. Differential Geom. 6 (1971), 119-128.

[EG] J.-H. Eschenburg and G. J. Galloway, Lines in spacetimes, Comm. Math. Phys. 128 (1992), 209-216.

[E] J.-H. Eschenburg, The splitting theorem for space-times with strong energy condition, J. Differential Geom. 27 (1988),.477-491.

[EH] J.-H. Eschenburg and E. Heintze, An elementary proof of the Cheeger-Gromoll splitting theorem, Ann. Global Anal. Geom. 2 (1984), 141-151. 
[G1] G. J. Galloway, A generalization of the Cheeger-Gromoll splitting theorem, Arch. Math. (Brno) 47 (1986), 372-375.

[G2] _ The Lorentzian splitting theorem without the completeness assumption, J. Differential Geom. 29 (1989), 373-389.

[LT] P. Li and L. F. Tam, Harmonic functions and the structure of complete manifolds, J. Differential Geom. 35 (1992), 359-383.

[L] Z. Liu, Ball covering on manifolds with nonnegative Ricci curvature near infinity, Proc. Amer. Math. Soc. 115 (1992), 211-219.

Department of Mathematics, University of Miami, Coral Gables, Florida 33124

Department of Mathematics, University of South Carolina, Columbia, South CarOLINA 29208 